\title{
Waiting times between gamma-ray flares of Flat Spectrum Radio Quasars, and constraints on emission processes
}

\author{
L. Pacciani ${ }^{1}$ \\ Istituto di Astrofisica e Planetologia Spaziali - Istituto Nazionale di Astrofisica (IAPS-INAF), Via Fosso del Cavaliere, 100 - I-00133 \\ Rome (Italy) \\ e-mail: luigi.pacciani@inaf.it
}

November 29, 2021

\begin{abstract}
Context. The physical scenario responsible for gamma-ray flaring activity and its location for Flat Spectrum Radio Quasars (FSRQ) is still debated.

Aims. The study of the statistical distribution of waiting times between flares (defined as the time intervals between consecutive activity peaks, see Wheatland \& Litvinenko 2002, for a formal description) can give information on the distribution of flaring times, and constrain the physical mechanism responsible for gamma-ray emission.

Methods. We adopt here a Scan-Statistic driven clustering method (iSRS, Pacciani 2018) to recognize flaring states within the FERMILAT archival data, and identify the time of activity peaks.

Results. We obtained that flares waiting times can be described with a poissonian process, consisting of a set of overlapping bursts of flares, with an average burst duration of $\sim 0.6$ year, and average rate of $\sim 1.3 \mathrm{y}^{-1}$.

For short waiting times (below 1d host-frame) we found a statistically relevant second population, the fast-component, consisting of a few tens of cases, most of them revealed for CTA 102. Interestingly, the period of conspicuous detection of the fast component of waiting-times for CTA 102 coincides with the crossing time of the superluminal $\mathrm{K} 1$ feature with the $\mathrm{C} 1$ stationary feature in radio reported in Jorstad et al. (2017); Casadio et al. (2019).

Conclusions. To reconcile the mechanism proposed in Jorstad et al. (2017); Casadio et al. (2019) with the bursting activity, we have to assume that plasma streams with a typical length of $\sim 2 \mathrm{pc}$ (in the stream reference frame) reach the recollimation shock.

Otherwise, the distribution of waiting times can be interpreted as originating from relativistic plasma moving along the jet for a deprojected length of $\sim 30-50 \mathrm{pc}$ (assuming a bulk $\Gamma=10$ ), that sporadically produces gamma-ray flares.

In magnetic reconnection scenario, reconnection events or plasma injection to the reconnection sites should be intermittent. Individual plasmoids can be resolved in a few favourable cases only (Christie et al., 2019); they could be responsible for the fast component.
\end{abstract}

Key words. galaxies: active — galaxies: jets — quasars: general — radiation mechanisms: non-thermal

\section{Introduction}

Blazars are the most luminous extragalactic objects observed in gamma-ray. They emit from Radio to TeV.

There is a general consensus that they are among the active galactic nuclei (AGN) that are able to produce a jet approximately oriented along the polar axis. In particular, blazars are jetted AGN for which the line of sight of the observer lies close to the jet axis (Urry \& Padovani 1995).

VLBA images of AGN jets at $15 \mathrm{GHz}$ show that jets geometry in the region from $10^{2}$ to $10^{3} \mathrm{pc}$ from the core is approximately conical with an aperture of $\sim 1^{\circ}$ (Pushkarev et al. 2017). Several jets have parabolic shape on shorter distances.

Relativistic features (Lister et al. 2016; Hovatta et al. 2009) moving downstream the jet are observed. They do not fill the entire cross-section of the ejection cone (Lister et al. 2013). Their motion is not always ballistic, showing radial and non-radial acceleration.

According to the Broad-Line emission power with respect to the optical continuum (Urry \& Padovani 1995), blazar are sub-divided in Flat Spectrum Radio Quasar (FSRQ, showing strong Broad Emission Lines) and BL Lac objects (with weak or absent Broad Emission lines).
The gamma-ray observed from blazars is due to the Doppler boosted emission from the jet.

The mechanism responsible for gamma-ray emission is still a debated issue. It is also controversial the kind of accelerated particles (leptons, hadrons) originating the gamma-ray emission, the accelerating engine, and its location.

Particles can accelerate through the shock diffusive acceleration (Aller et al. 1985; Hughes et al. 1985; Blandford \& Eichler 1987). In this model, piston-driven shock originates blazar outbursts; the shock compresses and orders the upstream magnetic field, causing the electron to accelerate though the Fermi mechanism, and the emitted synchrotron radiation to be polarized.

Alternatively, Narayan \& Piran (2012); Marscher (2013, 2014) proposed turbulence inside the jet as accelerating mechanism: flares are triggered by the passage of turbulent relativistic plasma flow in a re-collimation shock, located at parsec scale from the central supermassive black hole (SMBH). The shock compresses the plasma and accelerates electrons. The emission from single turbulent cells is responsible for rapid flares.

In magnetically dominated flows, magnetic reconnection driven by magnetic instabilities along the jet can efficiently accel- 
erate particles (Zenitani \& Hoshino 2001; Guo et al. 2014). Giannios (2013) found that magnetic reconnection events lead to the formation of plasmoids, whose emission overlaps in an envelope. Exceptional plasmoids can grow and produce fast flares that can be resolved from the envelope .

In leptonic models for FSRQs emission, the Inverse-Compton on an external target photon field (EC) is often adopted to explain the gamma-ray emission from relativistic electrons (Maraschi et al. 1992). Seed photons can be the continuum and line emission from the Broad Line Region, or the black body emission from dusty torus.

There is general agreement that if this is the emission mechanism, the flare peak-luminosity is proportional to the energy density of the external photon field (Dermer et al. 1997).

It is also found that electrons radiating via Inverse-Compton mechanism on external photon field dissipate their kinetic energy with a cooling time which is inversely proportional to the energy density of the target field (see, e. g., Felten \& Morrison 1966).

Alternatively, synchrotron radiation produced during flares is the target photon field for inverse-Compton scattering (Synchro-Self Compton model, SSC, Maraschi et al. 1992; Marscher \& Bloom 1992). This model is often invoked to model the gamma-ray emission of BL Lac objects.

Protons accelerated within the jet could reach the threshold for photo-pion production. In this model, the proton-synchrotron, the $\pi_{0} \rightarrow \gamma \gamma$ decay are responsible for jet gamma-ray emission (hadronic model, see. e.g. Bottcher 2013).

Casadio et al. (2015); Casadio et al. (2019) showed that several gamma-ray flares of FSRQs can be associated in time with superluminal features crossing stationary features along the jet observed at $43 \mathrm{GHz}$. They associated the stationary feature $\mathrm{C} 1$ that they observed in the jet of CTA 102 with a recollimation shock. In particular, Casadio et al. (2019) argued that the interaction of the superluminal structure $\mathrm{K} 1$ with recollimation shock $\mathrm{C} 1$ generates gamma-ray flaring activity from the end of 2016 to the beginning of 2017.

Larionov et al. (2020) discussed a similar scenario for 3C 279 observed from radio to gamma-ray for the years 2008-2018. They indeed noted that the evaluation of crossing-time of radio steady features by superluminal features have large uncertainties, thence the association of crossing-time with gamma-ray activity had a large chance to be spurious.

In this paper we study flaring activity in gamma-ray of FSRQ contained in the third Fermi-LAT catalogue (3FGL, Acero et al. 2015). In particular, we focus on waiting times between activity peaks, defined as the time interval $\Delta_{t}$ between two consecutive activity peaks (see Wheatland \& Litvinenko 2002, for a description of waiting times). The study is performed over $9.5 \mathrm{y}$ of data, from the beginning of the FERMI-LAT operation Atwood et al. 2009), up to the failure of a solar panel actuator. During this period of whole-sky survey, the monitoring with FERMI-LAT can be considered continuous, with the satellite scanning each region of the sky approximately every two-four orbits. Other authors (see, e.g., Abdo et al. 2010; Nagakawa \& Mori 2013; Sobolewska et al. 2014; Meyer, Scargle \& Blandford 2019; Tavecchio et al.. 2020) studied blazar variability in gamma-ray, focusing on gamma-ray light curves, rather than on waiting time between activity peaks. We, instead, disentangle the investigation of gamma-ray variability in a study of a temporal distribution of waiting times (this paper), and a study of correlated distribution of peak luminosity and flare duration (discussed in a forthcoming paper).

It's useful to report here some useful distribution concerning waiting times: suppose to have a uniformly distributed sample of events within a period of duration $\mathrm{T}$; the distribution of $\Delta_{t}$ between consecutive events inside the period is exponential $\rho=\frac{1}{\tau} e^{-\frac{\Delta_{t}}{\tau}}$ (where $\tau$ is the mean waiting time between consecutive events). Moreover, the time interval $\Delta_{t}=t_{i+k}-t_{i}$ between the events $i$ and $i+k$ of the time-ordered list of events is Erlang distributed with probability distribution function $\rho=\frac{1}{\tau^{k} K !} \Delta_{t}^{k-1} e^{-\frac{\Delta_{t}}{\tau}}$. If, for some reason, we do not detect some event, the observed distribution of waiting times between detected events deviates from an exponential distribution. For very short waiting times $\left(\Delta_{t} \rightarrow 0\right)$, indeed, the exponential trend is restored, because the contribution of the Erlang distribution for $k>1$ tends to 0 .

\section{2. analysis method}

We investigated the FERMI-LAT archival data, prepared data using the standard Fermi Science Tools (v10r0p5), and used the PASS8 Response Functions. We applied standard analysis cuts, and selected SOURCE class events (evclass $=128$ ) within $20^{\circ}$ from the investigated source using gtselect. We applied a zenith angle cut of $90^{\circ}$ to reject Earth limb gamma-rays.

We identified activity periods of each source using the iSRS clustering method (Pacciani 2018) applied to 9.5 years of gamma-ray data.

It is useful to report some definition and the method used in Pacciani (2018): For each source an extraction region is chosen for every event, it corresponds to the $68 \%$ containment radius (the containment radius depends on Energy and event type). Gamma-ray events within the chosen extraction region around the source catalogue position are selected. We can evaluate the cumulative exposure of the instrument to the source from the start of the FERMI-LAT operation to the time of arrival of each gamma-ray photon: gamma-ray events are time tagged, and also (cumulative) exposure tagged. Clusters of events are obtained applying the $i S R S$ procedure in the cumulative exposure domain, not in time domain (this is a reasonable aspect because the exposure to the source varies with time). From the dataset of each source, the set of clusters obtained with the $i S R S$ procedure are statistically relevant (we used a threshold for chance probability $P_{t h r}=1.3 \times 10^{-3}$, and a tolerance parameter $\left.N_{\text {tol }}=50\right)$. This set is a tree, called unbinned light curve. The clusters representing the activity peaks are the leaves of the tree. For each leaf, a peak time, and peak flux are identified. The branches of the tree ending with a leaf are the detected flares of the unbinned light curve: detected flares are described by a branch that contains at least the leaf.

As an example, we report in fig. 11 the unbinned light curve for PKS 1510-08. The horizontal segments are the relevant clusters of events obtained with the $i S R S$ procedure. They are described by their starting time, duration, and by the density of events in the exposure domain, e.g., by the average photometric source flux for the period of time subtended by the cluster. As far as the background is not subtracted, the flux is denoted with $F_{S R C+B K G}$. Vertical bars represent the error on flux estimate. Clusters are organized with a tree hierarchy. Each cluster is statistically relevant with respect to its parent: assuming that events in a parent cluster are uniformly distributed, there is a probability $\leq P_{t h r}$ that the son cluster is obtained by chance from the parent cluster. The cluster at the base of the tree is the root.

We define fully resolved a flare for which the branch contains 


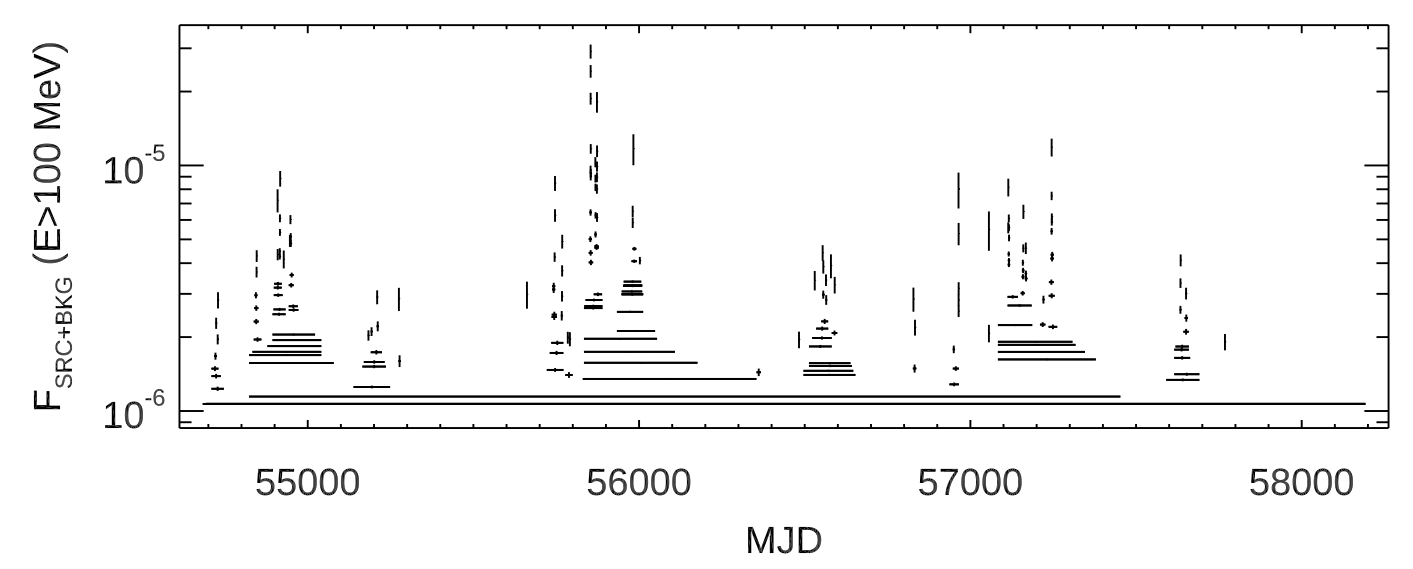

Fig. 1. Unbinned light curve (Pacciani 2018) for PKS 1510-08, obtained with $P_{t h r}=1.3 \times 10^{-3}$. Horizontal lines are the statistically relevant clusters. The set of statistically relevant clusters is a tree, and a hierarchy among clusters exists. For each son cluster there is a probability $\leq P_{t h r}$ to be obtained starting from its parent cluster by chance. Vertical bars are the errors on flux estimate. Background is not subtracted.

the leaf and at least an other cluster, and we define resolved a flare for which the branch contains the leaf alone.

Photometric flux at peak $F_{\text {phot }}^{\text {peak }}$ was extracted for resolved flares. Photometric flux at peak $F_{\text {phot }}^{\text {peak }}$ and flare duration $\left(F W H M^{\text {flare }}\right.$, full width half maximum) were extracted for fully resolved flares.

We also applied the standard analysis tools to extract flux ( $F_{\text {like }}^{\text {peak }}$, based on likelihood method) and its significance (from TS statistic) of the source for the periods of activity identified by the leaves.

All photometric methods suffer positional pile-up due to the instrumental Point Spread Function (PSF) and the presence of nearby sources. The method adopted here to study source variability is photometric, and it is sensitive to variability of the positional pile-up (spurious flares). To disentangle spurious flares, we classified as spurious, and coming from contiguous sources, flares with a ratio $R_{\text {photo2like }}=\frac{F_{\text {phot }}^{\text {peak }}}{F_{\text {like }}^{\text {peak }}}>1.3$, or with $T S<9$.

with this method, we prepared the sample used in this study. There will remain some contamination in the sample from spurious sources; and some systematic could affects the results that we will show. We account for the systematic effects by comparing the results varying the threshold on $R_{\text {photolike }}$ from 1.1 to 2 .

\section{Samples of activity peaks}

We selected gamma-ray emitting FSRQs, starting from the $3^{\text {rd }}$ FERMI-LAT catalogue (3FGL). We identified gamma-ray flaring periods from the FERMI-LAT archival data, within the period 2008 August - 2018 February.

As far as the gamma-ray analysis of sources at low galactic latitude is a cumbersome task, especially if photometric methods are adopted, we restrict the investigation to the high latitude FSRQs $\left(b>15^{\circ}\right)$ contained in the third Fermi-LAT catalogue. To avoid contamination from spurious sources within the 3FGL, we took into account for bright catalogue sources, with a TS statistic $\geq 49$. With these restrictions, we investigated 335
FSRQs.

We obtained two samples of activity peaks, based on the energy range of gamma-ray photons: A first sample is obtained selecting gamma-ray events with an energy $E>300 \mathrm{MeV}(300$ MeV sample);

Starting from the sources with at least 2 flares in the $300 \mathrm{MeV}$ sample, we also prepared a sample of activity peaks from the gamma-ray events with $E>100 \mathrm{MeV}$ (100 MeV sample).

To resolve in time two close flares, large statistics is needed (Pacciani 2018). In Appendix C, we show the effect on simulated samples. For this reason, in this paper we make use of the $100 \mathrm{MeV}$ sample.

\section{Results}

In this paper we always report waiting times, rates and duration in the host galaxy reference frame, except where explicitly written.

We performed several uniformity and unimodality tests on the sets of activity peaks of the apparently loudest sources (the sources with the largest number of detected flares). They are reported in Table 1 In the assumption that the FERMI-LAT regularly scanned each sky position, the distribution of flaring times cannot be considered neither uniform, nor unimodal.

The obtained distribution of waiting times (with logarithmic bin) is shown in Figure 2 (left column), while in the right column is reported the same distribution divided for the binsize; the last distribution is proportional to $\rho\left(\log \left(\Delta_{t}\right)\right) e^{-\Delta_{t}}$. Fitting functions are superimposed to the data. The fitting function reported with dashed line represents a set of overlapping bursts of flares (the multi-loghat distribution described in appendix A). The parameters of the fitting functions are reported in Table 2] The multi-loghat distribution is a poissonian process: the events within a burst are uniformly distributed. There is good agreement between data and the multi-loghat model for $\Delta_{t}$ larger than 1 day. For short waiting times $\left(\Delta_{t} \rightarrow 0\right)$ the multiloghat distribution and all the distributions based on Poisson processes (see, e.g., Wheatland 2000) shows an exponential profile $\rho\left(\Delta_{t}\right) \rightarrow \frac{1}{\tau} e^{-\frac{\Delta_{t}}{\tau}}$ (where $\tau$ is the typical timescales of 
Table 1. Tests for time distribution of flares on sources showing $N_{\text {flares }}>15$ in the $100 \mathrm{MeV}$ sample: Kolmogorov-Smirnov test of uniformity (K-S Kolmogorov 1933), Frosini test of uniformity (Frosini 1987), Hartigan \& Hartigan test of unimodality (Hartigan \& Hartigan 1985). The test value $\left(\mathrm{D}_{K S}, \mathrm{~B}, \mathrm{D}_{H H}\right)$ are reported for Kolmogorov-Smirnov, Frosini, and Hartigan \& Hartigan tests respectively. The probability (p) is also reported for the null hypothesis of uniformity or unimodality.

\begin{tabular}{lcll}
\multirow{2}{*}{ source } & \#flares & \multicolumn{2}{c}{$\mathrm{K}-\mathrm{S}$} \\
\cline { 3 - 4 } & & $\mathrm{D}_{K S}$ & $\mathrm{p}$ \\
PKS 1510-08 & 39 & 0.208 & 0.058 \\
3C 454.3 & 37 & 0.319 & $7.5 \times 10^{-4}$ \\
CTA 102 & 34 & 0.593 & $6.7 \times 10^{-6}$ \\
3C 279 & 28 & 0.634 & $9.0 \times 10^{-14}$ \\
4C +21.35 & 18 & 0.379 & $7.7 \times 10^{-3}$ \\
4C +71.07 & 17 & 0.327 & 0.040
\end{tabular}

the distribution). Thence, the fitting distribution reported in the right column of Figure 2 tends to a constant. Experimental data show this trend (Figure 2), but for $\Delta_{t}<1 d$ data deviate from the typical Poissonian profile, and show an increasing trend for reducing values of $\Delta_{t}$.

We can regard the distribution as combined of two distributions: a multi-loghat distribution and an other one acting on short waiting times (see, e.g., Aschwanden \& McTiernan 2010, for the study of solar flares with ISEE-3/ICE). We call fast component the second distribution; and we call multi log-hat + poissonian the composite distribution. Only a few tens of waiting times can be ascribed to the fast component, thence we cannot establish a reliable functional form with this dataset. We simply added a further poissonian term responsible for short waiting times. The resulting fitting function for the whole data sample is shown in Figure 2 (top row) and its parameters are reported in Table 2 The observed fraction of short waiting times is reported as $R_{\text {fast observed }}$ in Table 2. The reduction of the Cash estimator obtained adding the fast component tells us that the fast component is statistically relevant.

We tested also other fitting functions for the temporal distribution of flares. We obtained an intermediate result in term of Cash estimator with the multi-pow distribution function (defined in appendix A). Results are reported in Table 2. The multi-pow distribution, indeed, does not reproduce the trend observed for $\Delta_{t}<1 d$. It gives better results with respect to the multi log-hat distribution, because for the multi-pow distribution, the function $\rho\left(\log \left(\Delta_{t}\right)\right) e^{-\Delta_{t}}$ rises while $\Delta_{t}$ decreases. Thence, it roughly reproduces data with short $\Delta_{t}$.

We also tried to fit with another two-components distribution: the multi-pow + poissonian (defined in appendix A, with 5 parameters). Results are reported in Table 2 With this distribution we obtained the best Cash-estimator, but it is statistically comparable with the value obtained with the multi-loghat + poissonian. The multi-loghat+poissonian and the multi-pow distributions (with 5 parameters) are the statistically relevant models: in fact, they give $\Delta C=-10.0$, and $\Delta C=-11.5$ respectively with respect to the multi-pow distribution (with 3 parameters).

We have shown in appendix $\mathrm{C}$ that the resolving power depends on the temporal distance between flares, and on peak fluxes. Moreover, the apparently loudest sources are also the brightest ones. Thence we expect that the apparently loudest sources show the largest amount of short waiting times. This trend shows up in Figure 2 .

We will show for a physical scenario that close flares could not be resolved individually (pile-up effect), causing a reduction of

\begin{tabular}{lllll}
\multicolumn{2}{c}{ Frosini } & & \multicolumn{2}{c}{ Hartigan } \\
\cline { 1 - 2 } \cline { 5 - 6 } 0.514 & 0.089 & & 0.0756 & 0.070 \\
0.634 & 0.031 & & 0.082 & 0.045 \\
1.80 & $2.2 \times 10^{-16}$ & & 0.0698 & 0.20 \\
0.884 & $1.5 \times 10^{-3}$ & & 0.0997 & 0.020 \\
0.779 & $5.8 \times 10^{-3}$ & & 0.132 & $4.9 \times 10^{-3}$ \\
0.614 & 0.035 & & 0.131 & $7.9 \times 10^{-3}$
\end{tabular}

short waiting time statistics.

\section{1. a case study: CTA 102}

We note that the majority of short waiting times $\left(\Delta_{t}<1 d\right)$ come from CTA102. They are mainly grouped around MJD 57738 and MJD 57752. The unbinned light curve of the source for a $100 \mathrm{~d}$ period (obtained with a chance probability threshold $P_{t h r}=1.3 \times 10^{-3}$ ) is reported in fig 4 . The peculiar waiting times distribution is reported in Figure 3 .

The unbinned light curve can be compared with the results reported in D'Ammando et al. 2019 and in Meyer, Scargle \& Blandford 2019. There are 2 fast flares that were fully resolved with the $i S R C$ : the first flare peaks at MJD 57752.50 with a duration of 7.8 hours (FWHM, observer frame), the second one peaks at MJD 57752.83 with a duration of 1.5 hours (FWHM, observer frame). A further fast flare is fully resolved adopting a $P_{t h r}=2.3 \%$ : peaking at MJD 57738.00 , with a duration of 2 hours. We further note that in the $0.3 \mathrm{~d}$ period between MJD 57738.2 and MJD 57738.5 the $i S R S$ procedure resolves a set of 4 peaks (but the statistics does not allow to fully resolve flares). Thence, the average duration of each flare of the set is $\leq 0.1 \mathrm{~d}$. Similarly, in the $1 \mathrm{~d}$ period between 57751.8 and 57753.0 there are 3 resolved and 2 fully resolved flares, for an average flare duration of $\leq 0.2 \mathrm{~d}$.

We discuss in appendix $C$ the temporal pile-up. In particular flares with a temporal distance of $0.1 \mathrm{~d}$ could be resolved if their flux exceed $2 \times 10^{-5} \mathrm{ph} \mathrm{cm}^{-2} \mathrm{~s}^{-1}$ (Figure 11). This is the case for the resolved flares just mentioned. We cannot exclude that the tail preceding the peak at MJD 57738.00 is due to the pile-up of several flares with a trend of decreasing waiting times, or a trend of increasing flux. In fact, there is an hint of a complex structure from the $12 \mathrm{~h}$ binned light curve reported in D'Ammando et al. 2019.

\section{2. a peculiar source: $S 40218+35$}

The sample of sources we analysed includes also S4 0218+35, a blazar with a gravitational lens along the line of sight. The effect of the gravitational lens shows up in the distribution of waiting times reported in Figure 5. They cluster around $\Delta_{t} \sim 11 \mathrm{~d}$ (observer frame), in close agreement with the waiting time of delayed echo measured with VLA (Biggs 1999; Cohen et al. 2000) and with the gamma-ray data itself (Barnacka et al. 


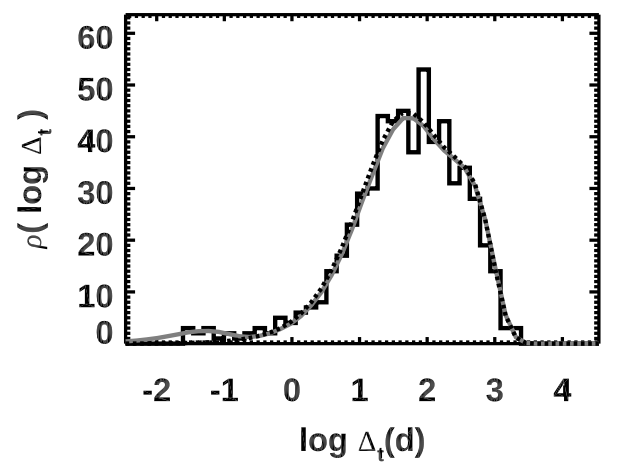

\section{all flares}
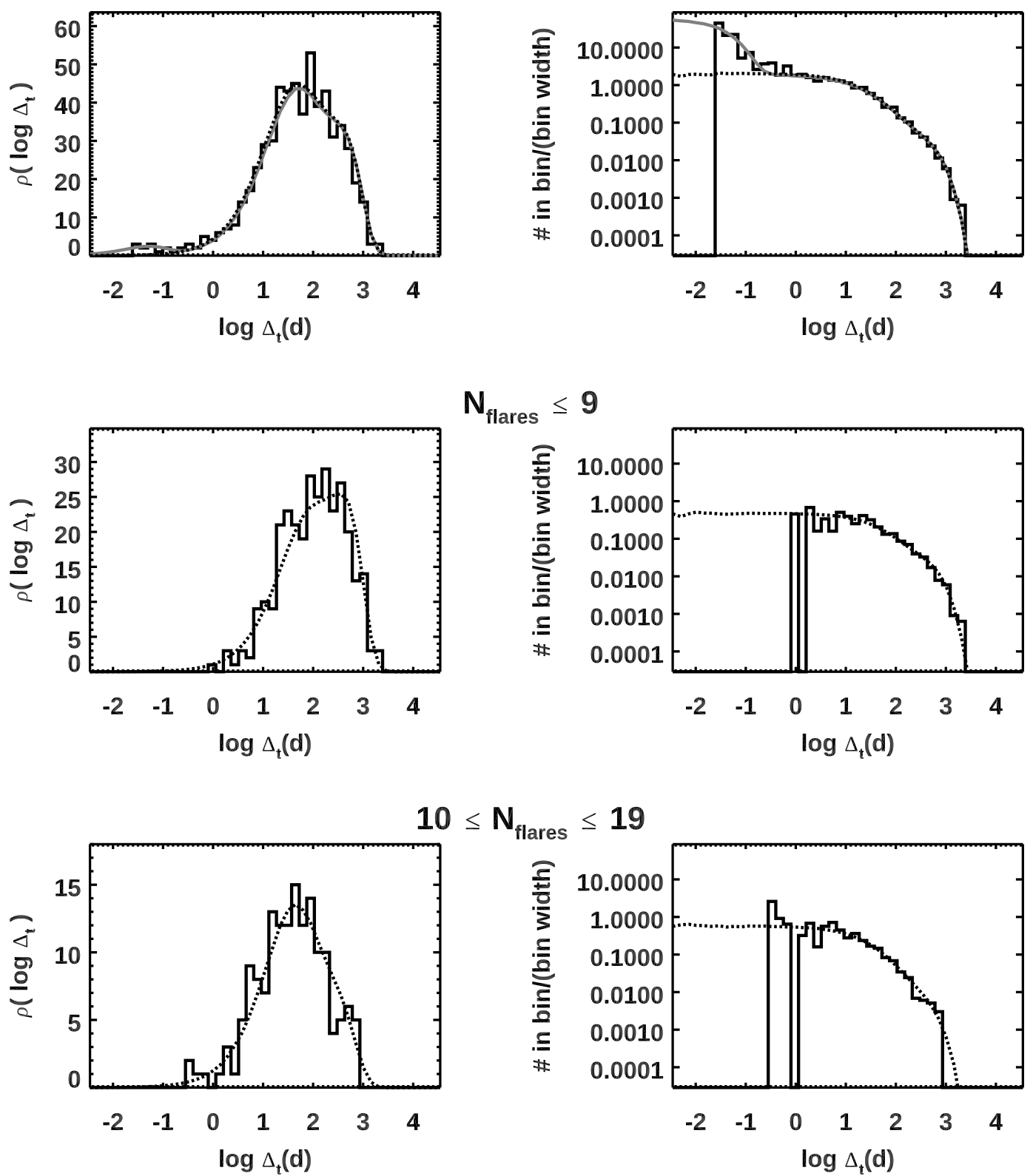

$10 \leq \mathbf{N}_{\text {flares }} \leq 19$

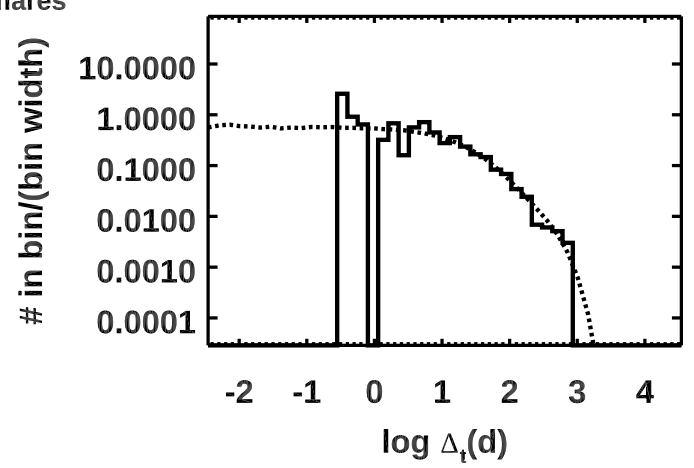

$\mathbf{N}_{\text {flares }} \geq 20$
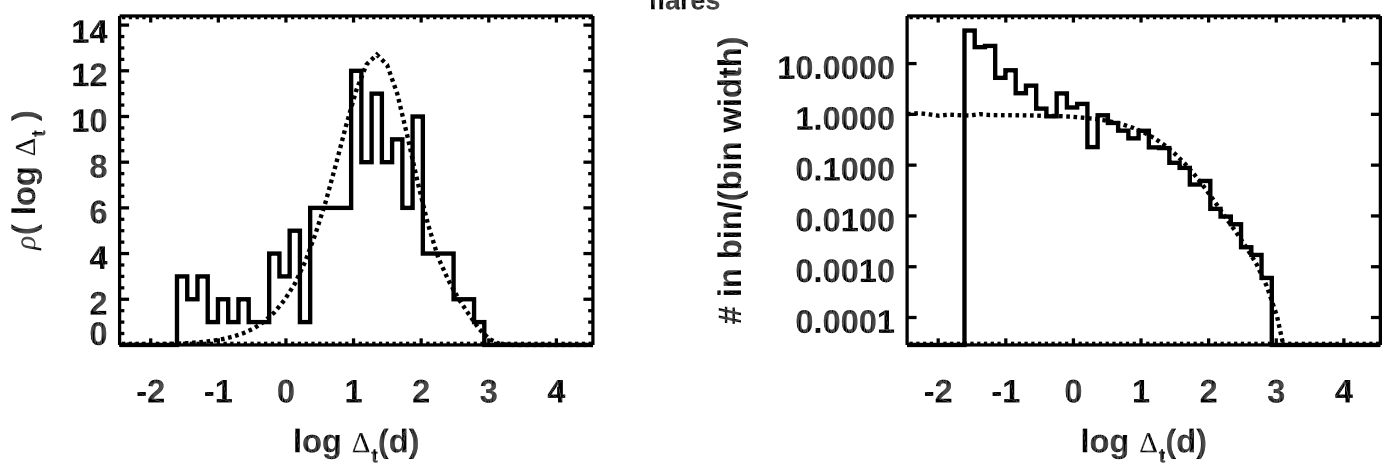

Fig. 2. Distribution of waiting times between gamma-ray flares of FSRQ detected with the iSRS method (100 MeV sample): Left columns report the distribution $\left(\rho\left(\log \left(\Delta_{t}\right)\right)\right.$ of $\log \left(\Delta_{t}\right)$. Right columns report the same distribution divided by the binsize (this new distribution is proportional to $\rho\left(\log \left(\Delta_{t}\right)\right) e^{-\Delta_{t}}$. The top row reports the distribution for all the sources. The other rows report the distribution of waiting times for sources selected on account of detected flares: from top to bottom $N_{\text {flares }} \leq 9,10 \leq N_{\text {flares }} \leq 19, N_{\text {flares }} \geq 20$. Solid histograms represent the data, dashed curves the multi-loghat fitting function. The solid grey line in the top row is the composite model obtained adding a poissonian process to the multi-loghat fitting function. 

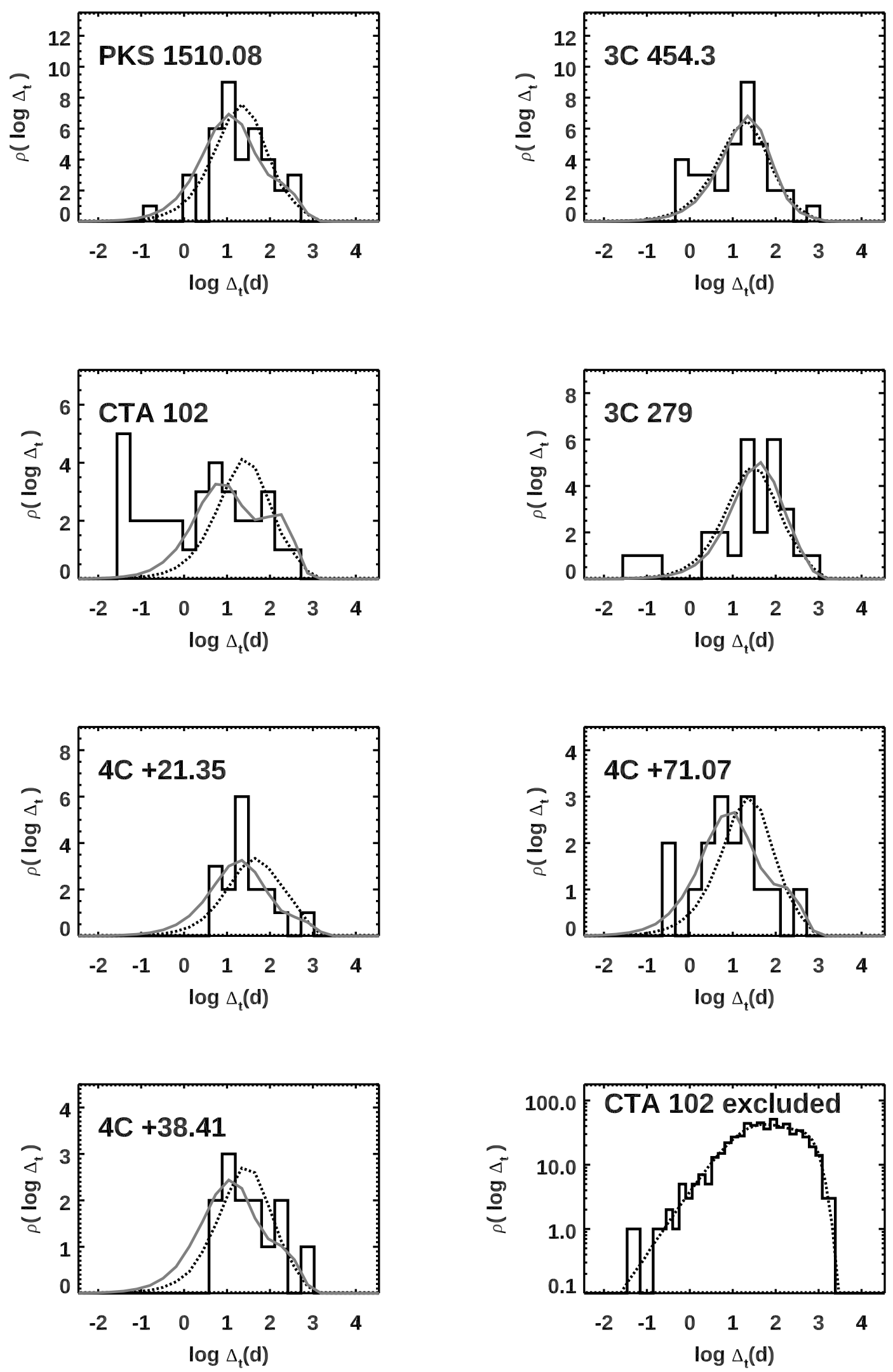

Fig. 3. Distribution of waiting times for the apparently loudest sources in the sample, and for the whole sample (excluding CTA 102, right bottom plot). Solid histograms represent the data, dashed curves the multi-loghat fitting function evaluated for the whole data sample. For single sources, the grey lines represent the multi-loghat fitting function evaluated for each source separately, with parameters reported in Table 3 , fit is performed for $\Delta_{t}>0.3 \mathrm{~d}$ to reduce the contribution of the fast component. Article number, page 6 of 15 

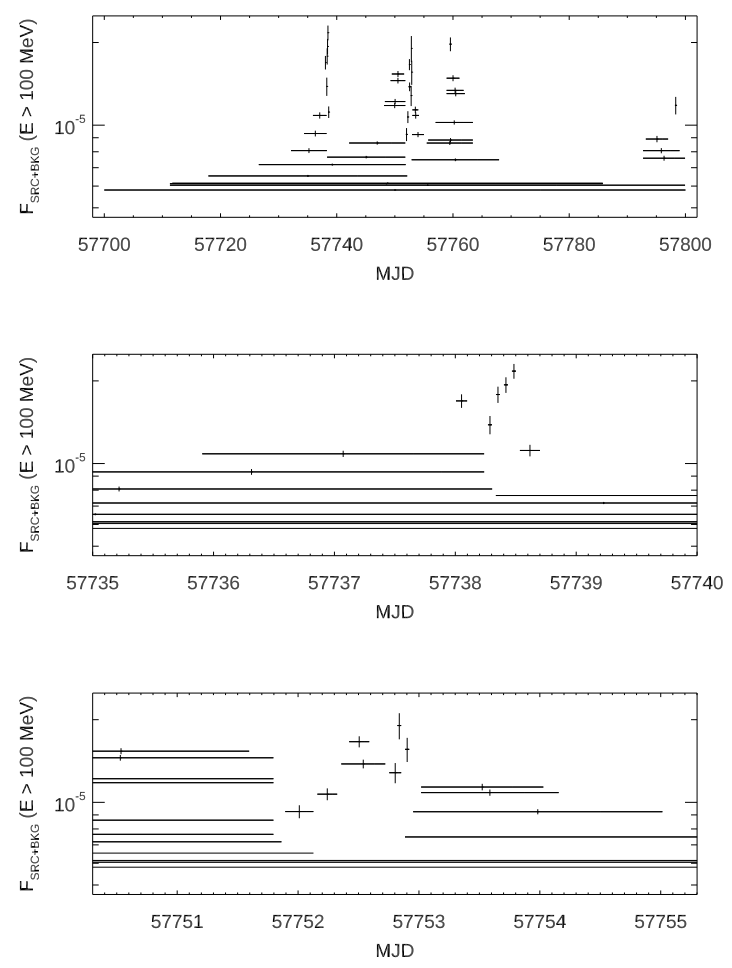

Fig. 4. Unbinned light curve (Pacciani 2018) for CTA 102 obtained for $\mathrm{E}>100 \mathrm{MeV}$ (see Pacciani 2018, for a detailed explanation), and zooms of relevant periods. The horizontal segments represent statistically relevant clusters of events obtained with the $i S R S$ procedure. They are described by duration, average photometric flux, and median time (or by starting time). Vertical lines are the error bars on flux estimate. The set of clusters form a tree called unbinned light curve. Background is not subtracted

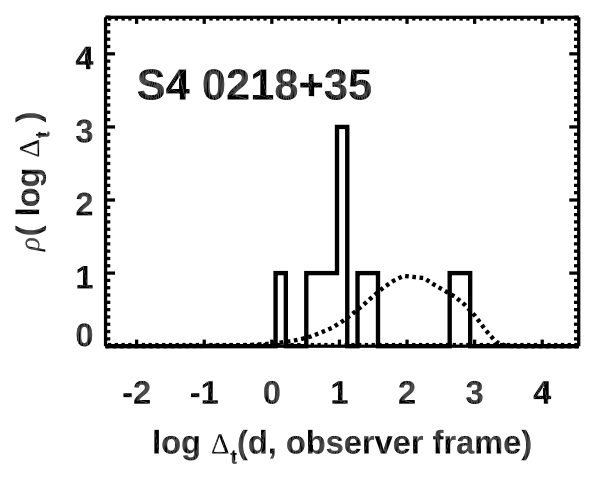

Fig. 5. Distribution of waiting times for the lensed source S4 $0218+35$ (observer reference system). Solid histogram represents the data, dashed curve the fitting function

\section{6).}

\section{3. remaining sources}

In order to reduce the signal from the short component, we performed a further investigation of waiting times distribution re-
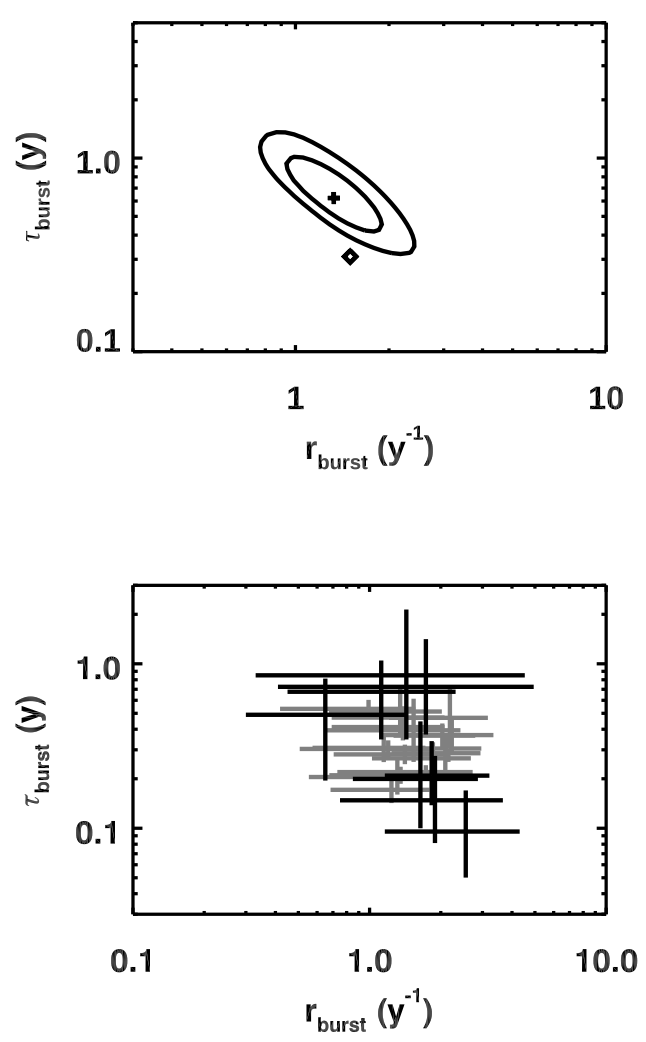

Fig. 6. Top panel: multi-loghat fit of waiting times for the $100 \mathrm{MeV}$ sample: joint confidence region for $r_{\text {burst }}$ and $\tau_{\text {burst }}$. The parameters for the minimum of the Cash estimator are reported with a cross. Contours are reported for $90 \%$ and $99 \%$ confidence levels. The resolved superluminal features ejection rate and fading time observed at 43 $\mathrm{GHz}$ (Jorstad et al. 2017) are reported with a diamond symbol (average values). Bottom panel: $r_{\text {burst }}$ and $\tau_{\text {burst }}$ (represented with black data) obtained by fitting the multi-loghat distribution to the data of single sources (for the apparently loudest sources in our gamma-ray sample). For comparison, the apparent ejection rate and fading times for each FSRQs reported in (Jorstad et al. 2017) are reported too (grey data).

moving CTA 102 from the sample.

We evaluated several fitting functions. The relevant are described in appendix A. In Figure 2 we show the fit with the multi-loghat distribution. It gives a satisfactory description of data; results are reported in Table 2, the joint confidence region for burst rate and duration parameters is reported in Figure 6 for the multi-loghat distribution (top panel). Fitting the multi-loghat function to the waiting time distribution generates correlated estimates of the two parameters.

The Cash estimator variation from an uniform to a multi-loghat function is $\Delta C=-105.8$, in favour of the multi-loghat distribution. We tested also other fitting functions for the temporal distribution of flares. We obtained slightly better results with the multipow distribution function. Results are reported in Table 2. Furthermore, we obtained that if fitting is limited to $\Delta_{t}>1 \mathrm{~d}$, Cash estimators for multi-loghat and multi-pow distribution become very similar.

Changing the pole position into the middle of the burst for the multi-pow does not produce a better value of the Cash estimator. Moreover, using multi-pow-smooth instead of a multi-pow does not give better Cash estimator. In Figure 3 we show the waiting 
Table 2. Fitted values of the parameters of the multi-loghat, multi-loghat+poissonian, multi-pow, multi-pow+poissonian distributions for the temporal distribution of flares (reported statistical errors with $90 \%$ confidence level, systematic errors are reported when they comparable to statistical errors). $C$ is the value of the Cash estimator at minimum. For multi-loghat model: $r_{\text {burst }}$ is the burst rate in $y^{-1}, m_{\log (\tau)}$ and $\sigma_{\log (\tau)}$ are the mean and standard deviation of the gaussian distribution for $\log \left(\tau_{\text {burst }}\right)$, with $\tau_{\text {burst }}$ in $y$. For multi-loghat + poissonian: the additional parameters are the fraction of events due to the fast component $\left(R_{\text {fast observed }}\right)$, and the typical timescale $\tau_{\text {fast }}$ of the fast component in hours, modelled with a poissonian process. For multi-pow model: $r_{\text {burst }}$ is the burst rate in $y^{-1}, \tau_{\text {burst }}$ is the burst duration in $y, \eta$ coefficient is described in appendix A. For multi-pow + poissonian: the additional parameters are the fraction of events due to the fast component $\left(R_{\text {fast observed }}\right)$, and the typical timescale $\tau_{\text {fast }}$ of the fast component in hours, modelled with a poissonian process.

multi-loghat (all sources)

\begin{tabular}{|c|c|c|c|}
\hline$C$ & $\begin{array}{l}r_{\text {burst }} \\
\left(y^{-1}\right)\end{array}$ & $m_{\log (\tau)}$ & $\sigma_{\log (\tau)}$ \\
\hline-2746.1 & $1.3 \pm 0.4$ (stat) & $-0.21_{-0.13}^{+0.18}($ stat $)$ & $0.36 \pm 0.14$ (stat) \\
\hline \multicolumn{4}{|c|}{ multi-pow (all sources) } \\
\hline$C$ & $\begin{array}{l}r_{\text {burst }} \\
\left(y^{-1}\right)\end{array}$ & -5 & $\begin{array}{l}\tau_{\text {burst }} \\
\left(y^{-1}\right)\end{array}$ \\
\hline-2766.0 & $1.5 \pm 0.3$ (stat) & $3.0_{-0.2}^{+0.5}(\mathrm{sys})_{-0.5}^{+0.4}(\mathrm{stat})$ & $0.67_{-0.00}^{+0.13}(\mathrm{sys})_{-0.16}^{+0.24}(\mathrm{stat})$ \\
\hline
\end{tabular}

multi-loghat + poissonian (all sources)

\begin{tabular}{|c|c|c|c|c|c|}
\hline C & $\begin{array}{l}r_{\text {burst }} \\
\left(y^{-1}\right) \\
13+03 \text { (stat) }\end{array}$ & $\begin{array}{l}m_{\log (\tau)} \\
-0.20^{+0.08}(\text { stat })\end{array}$ & $\sigma_{\log (\tau)}$ & $\begin{array}{l}\mathrm{R}_{\text {fast observed }} \\
(\%)\end{array}$ & $\begin{array}{l}\tau_{\text {fast }} \\
(h r)\end{array}$ \\
\hline-2776.0 & $1.3 \pm 0.3$ (stat) & $\begin{array}{l}-0.20_{-0.12}^{+0.08}(\mathrm{stat}) \\
\mathrm{ow}+\text { poissonian (all } \mathrm{s}\end{array}$ & $0.35 \pm 0.13$ (stat) & $2.9_{-0.11}^{+0.10}$ (stat) & $1.2_{-0.3}^{+0.1}(\mathrm{sys})_{-0.5}^{+1.0}$ (stat) \\
\hline$C$ & $\begin{array}{l}r_{\text {burst }} \\
\left(y^{-1}\right)\end{array}$ & $\eta$ & $\begin{array}{l}\tau_{\text {burst }} \\
\left(y^{-1}\right)\end{array}$ & $\begin{array}{l}\mathrm{R}_{\text {fast observed }} \\
(\%)\end{array}$ & $\begin{array}{l}\tau_{\text {fast }} \\
(\mathrm{hr})\end{array}$ \\
\hline-2777.5 & $1.4 \pm 0.3$ (stat) & $2.1_{-0.0}^{+0.4}($ sys $) \pm 0.5$ (stat) & $0.65_{-0.00}^{+0.09}(\mathrm{sys})_{-0.15}^{+0.22}$ (stat) & $2.5_{-0.0}^{+0.6}(\mathrm{sys})_{-1.0}^{+1.8}(\mathrm{stat})$ & $0.8_{-0.1}^{+0.2}(\mathrm{sys})_{-0.4}^{+1.3}(\mathrm{stat})$ \\
\hline
\end{tabular}

multi-loghat (CTA 102 excluded)

\begin{tabular}{|c|c|c|c|}
\hline$C$ & $\begin{array}{l}r_{\text {burst }} \\
\left(y^{-1}\right)\end{array}$ & $m_{\log (\tau)}$ & $\sigma_{\log (\tau)}$ \\
\hline-2642.1 & $1.3 \pm 0.4$ (stat) & $-0.21_{-0.13}^{+0.18}($ stat $)$ & $0.36 \pm 0.14$ (stat) \\
\hline \multicolumn{4}{|c|}{ multi-pow (CTA 102 excluded) } \\
\hline$C$ & $\begin{array}{l}r_{\text {burst }} \\
\left(y^{-1}\right)\end{array}$ & $\eta$ & $\begin{array}{l}\tau_{\text {burst }} \\
\left(y^{-1}\right)\end{array}$ \\
\hline-2643.8 & $1.4 \pm 0.3$ (stat) & $1.9_{-0.0}^{+0.4}(\mathrm{sys})_{-0.4}^{+0.6}(\mathrm{stat})$ & $0.64_{-0.00}^{+0.18}(\mathrm{sys})_{-0.10}^{+0.26}(\mathrm{stat})$ \\
\hline
\end{tabular}

times distribution of the apparently loudest sources in our sample, together with the multi-loghat fitting function, evaluated at minimum of the Cash estimator for the whole $100 \mathrm{MeV}$ sample, except CTA 102. A small amount of short waiting times are well outside the fitting distribution of single sources: the fast component arises also for sources other than CTA 102, but close in time activity peaks are resolved for extremely bright flares only. This is the case for 3C 454.3 flaring around MJD 55517-55521 and for 3C 279, flaring around MJD 57189.
The fast component does not show up in the distribution of waiting times accumulated for all the sources (except CTA 102). We will show that for realistic simulations (taking into account flare duration, and exposure variation with time), some pileup effect appears for $\Delta_{t}$ shorter than several days. The net effect is a reduction of statistics for short waiting times. We speculate that the cumulative distribution reported in Figure 3 (right bottom plot) does not show the pile-up effect, because the fast component compensates for the reduction of statistics for short waiting 
Table 3. Fitting parameters of the multi-loghat model for the temporal distribution of flares (reported statistical errors with 68\% confidence level). The first column reports the source name for which the fit was performed. The second column reports the value of the obtained minimum for the cash estimator. $r_{\text {burst }}$ is the burst rate in $y^{-1}, m_{\log (\tau)}$ is the mean of the gaussian distribution for $\log \left(\tau_{\text {burst }}\right)$, with $\tau_{\text {burst }}$ in $\mathrm{y}$. The standard deviation of the distribution of $\log \left(\tau_{\text {burst }}\right)$ is held fixed at the value obtained fitting the multi-loghat function to the accumulated distribution of waiting times $\left(\sigma_{\log (\tau)}=0.36\right)$. To keep at a minimum the contribution of the short waiting time component, fit is performed on the reduced sample with $\Delta_{t}>0.3 d$.

\begin{tabular}{|c|c|c|c|}
\hline source & cash & $r_{\text {burst }}$ & $m_{\log (\tau)}$ \\
\hline & & $\left(y^{-1}\right)$ & \\
\hline PKS 1510-08 & -43.3 & $1.8_{-0.7}^{+1.4}$ & $-0.68_{-0.18}^{+0.21}$ \\
\hline $3 C 454.3$ & -22.2 & $1.1_{-0.7}^{+1.2}$ & $-0.17_{-0.29}^{+0.19}$ \\
\hline CTA 102 & 5.4 & $2.5_{-1.4}^{+1.8}$ & $-1.02_{-0.28}^{+0.25}$ \\
\hline $3 \mathrm{C} 279$ & -3.2 & $1.7_{-1.3}^{+3.2}$ & $-0.14_{-0.29}^{+0.29}$ \\
\hline $4 C+21.35$ & -0.5 & $0.65_{-0.35}^{+0.78}$ & $-0.31_{-0.40}^{+0.22}$ \\
\hline $4 C+71.07$ & 10.1 & $1.9_{-1.1}^{+1.8}$ & $-0.83_{-0.26}^{+0.27}$ \\
\hline $4 C+38.41$ & 11.0 & $1.6_{-0.8}^{+1.2}$ & $-0.70_{-0.30}^{+0.35}$ \\
\hline PKS 0402-362 & 11.0 & $1.4_{-1.1}^{+3.1}$ & $-0.07_{-0.39}^{+0.40}$ \\
\hline
\end{tabular}

times.

In Table 3, in Figure 3 and 6 (bottom panel) we report the results obtained fitting the multi-loghat model to the waiting time distributions for single sources. Due to the limited sample size, we fitted model to the data of single sources fixing the parameter $\sigma_{\log (\tau)}$ to the value obtained by fitting the multi-loghat model to the accumulated distribution $\left(\sigma_{\log (\tau)}=0.36\right)$. Moreover, we restricted to $\Delta_{t} \geq 0.3 \mathrm{~d}$ to minimize the contribution of the fast component to the single source samples.

\section{Discussion and Conclusion}

\subsection{Waiting Times distribution for a non-ideal instrument}

In the previous section we studied the waiting time distribution for an ideal instrument. In order to investigate the effect of the exposure variation with time for each source, the gaps between observations of the FERMI-LAT, and the pile-up effect, we performed detailed simulations taking into account for the exposure to each source. We investigated three cases:

a) Flares uniformly distributed within the observation period of $9.5 \mathrm{y}$.

b) Time distribution of flares follows the multi-loghat distribution.

For both case $a$ and $b$ we extracted the logarithm of the Doppler factor of accelerated electrons $\log \left(\delta_{\text {flare }}\right)$ with a gamma distribution, with mean $=1$, and variance $=0.045$ (see the best-fit intrinsic jet properties obtained for the 1.5JyQC quasar sample in Lister et al. 2019). Flares duration is proportional to $\delta_{\text {flare }}^{-1}$.

The observer line of sight with respect to the jet axis, and the bulk Lorentz factor of the emitting feature affect the observed peak flux, flare duration, and waiting times between flares. The amount of the effect depends on the emission process responsible for gamma-ray emission, and on the physical scenario responsible for flaring activity. To account for this, we also studied a simple geometry for the emitting source. (case $c$ below):

$c$ Time distribution of flares follows the multi-loghat distribution: Flares are uniformly generated within the burst. Flares within each burst of activity have all the same Lorentz factors, and the same orientation (of the moving blob) with respect to the jet axis: it is uniformly extracted within a cone of aperture $1^{\circ}$ around the jet axis. The line of sight is uniformly extracted within a cone of $8^{\circ}$ aperture from the axis of the blazar jet. For each flare, peak luminosity and flare duration FWHM are anticorrelated. We explored two sub-cases: peak luminosity is proportional to $\delta_{\text {flare }}^{4+2 \alpha}$ (EC leptonic model, see, e.g. Dermer et al. 1997), and the FWHM is proportional to $\delta_{\text {flare }}^{-1}$ (light crossing time prevails on cooling, case $c_{l c t}$ ); or the flare duration FWHM is proportional to $\Gamma_{\text {flare }}^{-\frac{3}{2}} \delta_{\text {flare }}^{-\frac{1}{2}}$ (cooling time prevails, case $c_{\text {cooling }}$, see, e.g., Pacciani 2014).

The burst rate is a constant for all the sources. We didn't try to reproduce the fast component of waiting times, but only the multi-loghat component. This last case imposes that all the flares from the same burst have the same Doppler factor, and thence have a large coherence.

We modelled peak luminosity and flare duration of simulations with a parametric distribution, whose parameters were obtained by fitting the models to the peak-luminosity and duration of detected flares. Further details of the models will be described in a dedicated paper. For all the models, flare shape was modelled according to Equation 7 in Abdo et al. 2010, and converted in a temporal distribution of gamma-rays detected by an ideal telescope. We tried also to model the flare temporal profile with gaussian and gamma distributions, obtaining similar results. Finally each gamma-ray is randomly accepted or rejected according to the real exposure to the source. Exposure was evaluated for temporal bins of 86.4s. Once the time series were obtained for each source, we applied the $i S R S$ procedure to extract flares from simulated data (the same procedure used to extract flares from real data).

Regarding case $a$, we performed 100 simulations. We fitted the simulated waiting times distribution with both an uniform, both with a multi-loghat distribution. In fact the multi-loghat distribution approaches an uniform distribution for burst rates much larger than the flaring rate within a burst, and we can successfully fit the uniformly distributed samples using a multi log-hat fitting function. Due to the telescope exposure variation with time to each source, the final sample of recognized flares could mimic a multi loghat distribution of waiting times. We obtained for case $a$ (uniformly distributed flares) that the absolute variation of the Cash estimator from uniform to multi-loghat fitting function is $|\Delta C|<3$. We already found for real data that the variation of the Cash estimator from uniform to multi-loghat fitting distribution is $\Delta C=-105.8$. Thence the hypothesis that (for real data) uniformly distributed (in times) flares could mimics a multi-loghat like distribution of waiting times (due to exposure variation with time) is rejected. We found, instead, that fitting the multi-loghat function to the waiting time distributions obtained simulating case $b$ and $c$, the fitted bursting rate and duration corresponded to the simulated bursting rate, and duration of generated samples. So, the fitting procedure and results obtained fitting the multi-loghat to the data is validated with simulations. 


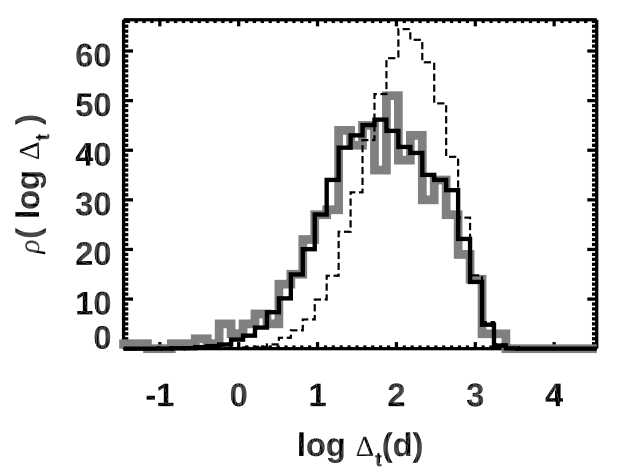

Fig. 7. Distribution of waiting times for data (grey line), the simulated case $a$ (uniformly distributed flares, thin dashed line), and simulated case $c_{l c t}$ (light crossing time prevails, black continuous line).

We note that for case $c$ the pile-up effect is reduced with respect to case $b$ : For case $c$ flares within the same burst are highly correlated, because have all the same Lorentz and Doppler factor.

From the detailed simulations we obtained that temporal resolving power reduces to $\frac{1}{2}$ at $\Delta_{t}=4 \mathrm{~d}, 2 \mathrm{~d}, 1.5 \mathrm{~d}$ respectively for case $b$, case $c_{\text {cooling }}$ (cooling time prevails), case $c_{l c t}$ (light crossing time prevails).

The detailed modelling cannot reproduce exactly the same number of detected flares, but we obtained that the revealed number of simulated flares for each source was at most within a factor $\sim 2$ with respect to real data. The comparison of the detailed simulation (case $c_{l c t}$ ) with the data is shown in Figure 7 The comparison was performed excluding CTA 102 from the $100 \mathrm{MeV}$ sample. The waiting times obtained from the simulated time series reproduce the behaviour of data. Some discrepancy remains for short waiting times $\left(\Delta_{t}<3 d\right)$ : In fact, with the detailed modelling, the pile-up effect shows up, suppressing the statistics for short waiting times. Moreover, the model does not try to reproduce the fast component acting on short timescales.

To summarize, a set of superimposing bursts of activity is able to reproduce the data. There is a suppression of statistic for short waiting times. Depending on the physical scenario, this suppression starts at $\Delta_{t} \sim 1.5-4$. The observed waiting time distribution for all the sources (CTA 102 excluded) reported in fig 2 apparently does not show suppression. We guess that the fast component compensates the pile-up suppression.

We could expect that gamma-ray FSRQs selected in this paper should have a broad distribution of the jet axis with respect to the line of sight, affecting the observed peak flux, flare duration, and waiting times between flares. As a results the accumulated distribution of waiting times should be broad too, due to the different viewing angles among sources, possibly washing out the multi-loghat time distribution proposed to fit the results. Instead, the flare luminosity dependency upon the doppler factor strongly mitigate the effect for flux limited samples: In fact flaring emitting regions, moving along a trajectory (that we identify with a flare direction) which is at large angle with respect to the line of sight, usually remain undetected in gamma-ray, because of the dependency of the flaring luminosity $\mathrm{L}_{\gamma}$ upon the doppler factor $\left(\mathrm{L}_{\gamma} \propto \delta^{4+2 \alpha}\right.$ for the external Compton, $\mathrm{L}_{\gamma} \propto \delta^{3+\alpha}$ for the synchrotron emission, see , e.g., Dermer et al. 1997). This suppression effect combines
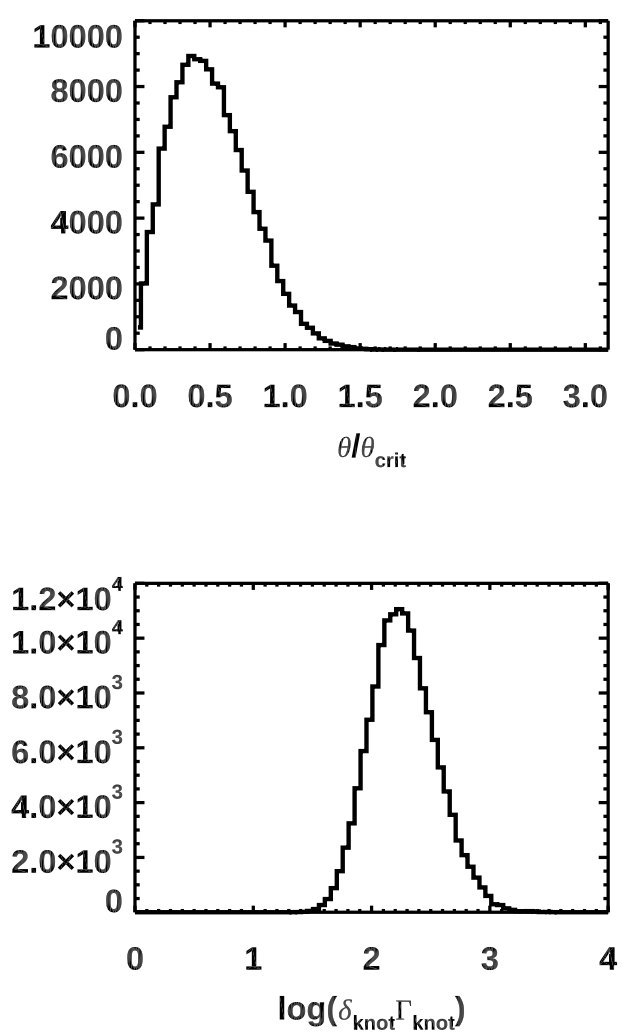

Fig. 8. Top panel: Distribution of viewing angles of gamma-ray detected flares obtained simulating case $c_{\text {cooling }}$. Bottom panel: Distribution of $\Gamma_{k n o t} \delta_{k n o t}$ for gamma-ray detected flares obtained simulating case $c_{\text {cooling }}$. To produce the histogram, the observer line of sight with respect to the jet axis was generated uniformly within a cone of aperture $30^{\circ}$.

with the low probability to observe flares from electrons with bulk motion aligned with the line of sight. As a result, viewing angles distribution for gamma-ray detected flares should be peaked at an intermediate value between 0 and the critical angle $\Theta_{c r}=\frac{1}{\Gamma_{b u l k}}$. The distribution of viewing angles of gamma-ray detected flares (obtained simulating the simplified scheme $c_{\text {cooling }}$ where the bulk direction of the flare emitting electrons coincides with the knot direction) is reported in figure 8 (top panel). This circumstance strongly reduces the broadening of the accumulated waiting time distribution. Suppression was investigated for flux-limited radio samples too, with jet viewing angle distribution peaking at about half of the critical angle (see, e.g., Vermeulen \& Cohen 1994; Lister \& Marscher 1997). Further suppression effect for gamma-ray detected blazars was also found in Savolainen 2010, and in Pushkarev et al. 2017, see their Figure 15, reporting the distribution of jet viewing angles of the Mojave Radio sample for Fermi-LAT detected and for Fermi-LAT not detected AGNs. Our sample shows strong suppression at large viewing angles, because it is obtained starting from Fermi-LAT detected FSRQs for which we revealed flaring activity. Thence the accumulated distribution of waiting times between flares that we studied here is obtained for sources with a narrow angle between the line of sight and the jet axis. The simulated cases $c_{l c t}$ and $\mathrm{c}_{\text {cooling }}$ show this suppression effect, and take into account for the non-monochromatic distribution of the bulk doppler factor of travelling knots (see Lister et al. 2019). 
In the case of uniformly distributed flares within a burst of activity, we expect for each source a waiting time distribution $\rho\left(\log \left(\Delta_{t}\right)\right) e^{-\Delta_{t}}$ with a flat tail for short waiting times. As a result, the accumulated distribution (obtained accumulating waiting times for all the sources) should still have a flat tail for short waiting times, irrespective of the differences of burst rate and duration among sources.

\section{2. constraints on gamma-ray emission models}

Jorstad et al. (2017), Casadio et al. (2019) argued that gammaray flares could be associated with superluminal moving features crossing stationary features along the jet. If this is the case, the burst activity (that we revealed from waiting times between activity peaks) could be associated with plasma streams relativistically moving along the jet, and crossing a steady feature along their path. Assuming a stream with $\Gamma_{\text {stream }}=$ 10 , its typical length (in its reference system) measured along the jet is $l_{\text {stream }}^{\prime}=\tau_{\text {burst }} \beta_{\text {stream }} c \Gamma_{\text {stream }} \sim 2 \mathrm{pc}$.

Alternatively, we could assume that a travelling perturbation or a travelling knot is responsible for the bursting activity. We could assume that in this case the direction of accelerated particles is highly correlated with the direction of the travelling knot (corresponding to case $c$ ). We can correlate the duration of the observed burst with the travelled length $l_{k n o t}$ of the superluminal feature:

$$
\tau_{\text {burst }}=\frac{l_{\text {knot }}}{\beta_{\text {knot }} c}\left(1-\beta_{\text {knot }} \cos \theta_{\text {view }}\right)=\frac{l_{\text {knot }}}{\beta_{k n o t} c \Gamma_{\text {knot }} \delta_{\text {knot }}}
$$

In this scenario the viewing angle of the jet axis varies from source to source, and the Lorentz factor of moving knots is not monochromatic. Adopting the proposed case $c$, the distribution of $\Gamma_{k n o t} \delta_{k n o t}$ for gamma-ray detected flares can be evaluated with simulations. It is reported in figure 8 (bottom panel) assuming the mean Lorentz factor for the moving feature is $\Gamma_{k n o t}=10$. We obtained a distribution of $\log \left(\delta_{k n o t} \Gamma_{k n o t}\right)$ with mean value $\sim 2.26$ and root mean square broadening $\sim 27 \%$; thence $l_{k n o t}$ is 30-50 pc. We underline that, on account of Equation 1 and of the spread of $\log \left(\delta_{k n o t} \Gamma_{k n o t}\right)$, the viewing angle variation from source to source, and the distribution of $\Gamma_{b u l k}$ could be, at least partially, responsible for the spread of $\tau_{\text {burst }}$ reported in Table 2 (parameter $\sigma_{\log (\tau)}$ of the multi-loghat distribution).

We can compare the achieved results with the VLBA observations reported in Jorstad et al. 2017. In their Table 7, they report the fading time $\tau_{43 \mathrm{GHz}}$ for moving features observed at $43 \mathrm{GHz}$. We report in Figure 9 the $\tau_{43 \mathrm{GHz}}$ distribution and emission rate for the resolved superluminal features observed at $43 \mathrm{GHz}$ (see Figures 4 in Jorstad et al. 2017). The mean value for $\log \left(\tau_{43 \mathrm{GHz}} / y\right)$ of the fitting logarithmic distribution is $-0.50 \pm 0.02$, and the standard deviation is $0.22 \pm 0.02$ respectively. It is tempting to associate the distribution of fading time of moving features, with the distribution of the duration of bursts of flares found in this paper and reported in Table 2. The comparison is reported in Figure 6. Our results agree with the knot emission rate and with the fading time (within a factor two) of knots observed at $43 \mathrm{GHz}$ (Jorstad et al. 2017).

As the knot travels away from the SMBH, we expect a significant reduction of peak flux of the emitted flares. In fact both the external magnetic field $B$, and the energy density of the external radiation field $u_{\text {ext }}$ decrease along the travelled path ( $B \propto \frac{1}{d}$, and $u_{e x t} \propto \frac{1}{d^{2}}$, with $d$ the distance of the emitting zone from the SMBH). Thence the peak flux of flares generated along the travelled path follows the decreasing trend with $d$ in optical (synchrotron emission) and in gamma-ray (assuming EC emission). We note indeed that the magnetic field reduction with the distance from the SMBH (and electron cooling) should affect also the fading time evaluated from radio luminosity of superluminal features (Jorstad et al. 2017). Homan 2015 showed that radio features accelerate for deprojected distances up to $\sim 100 \mathrm{pc}$ from the central SMBH. If flares are emitted while knots still experience acceleration, the enhancement of $\Gamma_{k n o t}$ could partially compensate the reduction of energy density of the external photon field.

Finally, in magnetic reconnection scenario (see, e.g., Giannios 2013), reconnection events can be triggered by magnetic instabilities such as kink instabilities (Begelman 1998; Spruit et A1, 2001), or magnetic field inversions at the base of the jet (Giannios \& Uzdensky 2019). We speculate that bursting activity we reveal could be generated by intermittent magnetic instabilities or by intermittent flux of plasma: instabilities phases of the magnetic structure of the jet, or the plasma injection to the reconnection sites should have a typical timescale of $\sim 0.6 y$.

Sironi, Giannios \& Petropoulou (2016) obtained that plasmoids can be produced continuously (plasmoids chain) if fresh plasma is added to the system. Due to the limited flare resolving power of the FERMI-LAT, we are rarely able to resolve plasmoid emission from the envelope (see, e.g., Christie et al.. 2019). Thence, the activity peaks we observe in gamma-ray correspond to the envelope emission from reconnection events. If this is the case, reconnection events must be uniformly produced in time during the development of magnetic instabilities. Moreover, we could associate the fast component to the rare cases for which we are able to resolve plasmoids within the envelope (when the jet axis and reconnection layer are almost aligned to the line of sight). In this physical scenario, the orientation of the reconnection layer differs for each reconnection event (such as in simulated case $b$ ), but plasmoids from the same reconnection event emit along the same direction.

\subsection{The Fast component and the gamma-ray data of CTA 102}

We observed a few tens of short waiting times (for waiting times $<1 \mathrm{~d}$ ), mainly detected for CTA 102. The typical timescale of this component is $\sim 1$ hour. The detailed simulations, performed taking into account for the FERMI-LAT exposure to the source, and realistic duration of flaring activity show that temporal pile-up of close-by flares acts for waiting times $<1.5-4 \mathrm{~d}$. We did not try to study the distribution of the fast component in more details with the simple fitting provided here. In fact pile-up plays a major role. Moreover the exposure to sources cannot be considered continuous for time periods below several hours. A study could be performed applying detailed modelling for particle acceleration and gamma-ray emission to the simulating chain.

A fast, white component, with similar timescale was found in optical for two BL Lac objects (Raiteri et Al. 2021a.b).

Interestingly, the fast component shows up in CTA 102 gammaray data for the time interval for which the superluminal component $\mathrm{K} 1$ crosses the $\mathrm{C} 1$ stationary feature of the jet, and for this event, the scenario of acceleration of turbulent cells from a recollimation shock was proposed (Casadio et al. 2019). Larionov et al. (2017) interpreted the CTA 102 period of activity differently: They argued that a superluminal knot 

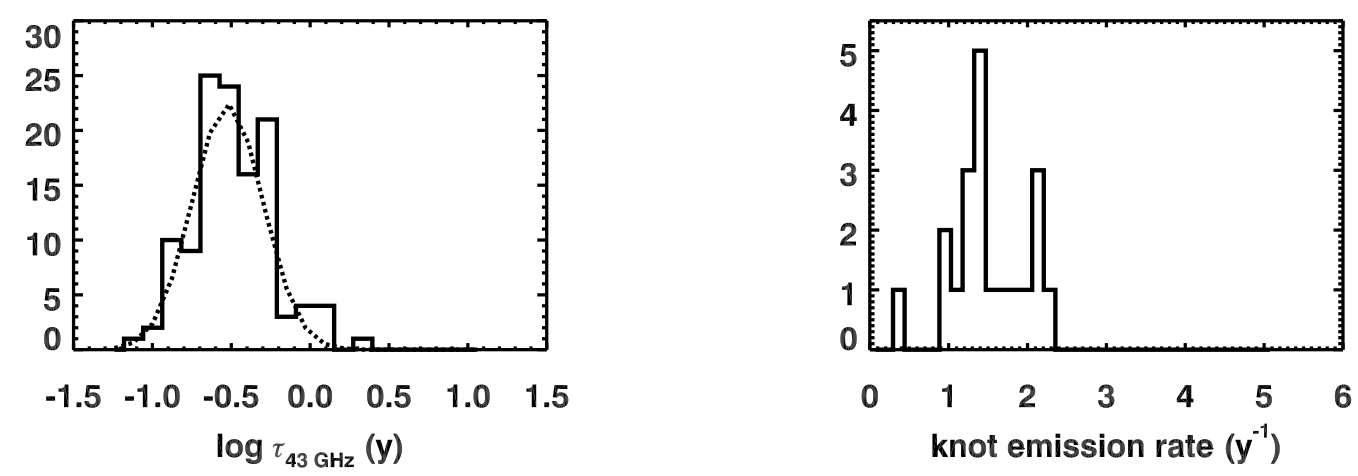

Fig. 9. Left panel: distribution of fading time of superluminal features observed at $43 \mathrm{GHz}$ (reported in Table 4 of Jorstad et al. 2017) (log scale). Right panel: distribution of resolved superluminal features ejection rate at $43 \mathrm{GHz}$ (from Table $5 \mathrm{of}$ Jorstad et al. 2017).

changing its own orientation and moving along the line of sight from the end of 2016 to the beginning of 2017 (during the period of conspicuous detection of the fast component) can produce the observed flux and polarization trend in optical and radio. We note that the change in orientation could explain the quadratic correlation observed in optical and gamma-ray (Larionov et al. 2017): In fact, it corresponds to a change in Doppler factor and then to a change in gamma-ray luminosity $L_{\text {gamma-ray }} \propto \delta^{4+2 \alpha}$ (with $\alpha$ the gamma-ray spectral index), and to a change in optical luminosity $L_{\text {optical }} \propto \delta^{3+\alpha}$, according to Dermer et al. (1997) for EC and synchrotron emission respectively. The weak polarization observed on 23-Dec-2016 reported in Casadio et al. (2019) for the K1 superluminal feature, witnesses for an aligned direction of the $\mathrm{K} 1$ component toward the observer.

The change in orientation scenario causes less than a factor 2 reduction in waiting times of gamma-ray, but it is responsible for a large increase of flux, and thence the activity peaks can be temporally resolved with the FERMI-LAT (we already discussed the net effect in the results for CTA 102). Thence, at least for a short period of time, we were able to resolve in gamma-ray the fast component flaring activity, due to turbulence, or to the plasmoid chain in reconnection scenario.

\section{Summary}

In this paper we found that gamma-ray activity peaks of FSRQs are produced in bursts. The average duration of a single burst is $\sim 0.6 \mathrm{y}$, and the average burst rate is $\sim 1.3 \mathrm{y}^{-1}$. These are averaged values obtained from the whole analysed sample. The temporal distribution of activity peaks within each burst can be approximated with an uniform distribution.

Our overall distribution of waiting times between activity peaks shows a statistically relevant fast component (with $\Delta_{t}<1 \mathrm{~d}$ ), that can be roughly modelled with an uniform distribution with a typical waiting time of $\sim 1 \mathrm{~h}$. CTA 102 shows the large majority of the fast population. Once CTA 102 is removed from the whole sample, the cumulative waiting time distribution of all the remaining sources does not show clear evidence of the fast population. Indeed, several cases of short waiting times, that can be ascribed to the fast component, can be found in the waiting time distribution of single sources. We evaluated the pile-up effect on the waiting time sample, and found that it must be relevant for waiting times shorter $1.5-4 \mathrm{~d}$, depending on the modelling. We guess that the fast component and the pile-up compensate each other in the cumulative distribution of waiting times of the remaining sources. Thence the fast component should act for waiting times shorter than $1.5-4 \mathrm{~d}$.

It's worth mentioning that Raiteri et Al. 2021ab found a statistically relevant white noise emerging for frequencies above $\sim 0.9 h^{-1}$ and $\sim 3.6 h^{-1}$ in the power spectral density analysis (PSD) of optical data of the BL Lac objects S4 0954+65 and S5 0716+714 respectively (observed with TESS, Transiting Exoplanet survey Satellite, Ricker et al. 2915, and WEBTI, Whole Earth Blazar Telescope organization, Villata et Al. 2002).

Our finding can be applied to existing theoretical/phenomenological models: (Narayan \& Piran 2012; Marscher 2013, 2014) proposed that gamma-ray flares originate from the passage of turbulent plasma inside a recollimation shock. In such a scenario, the burst of activity that we found could be associated with the length of a superluminal plasma stream. We estimated a length of $\sim 2 \mathrm{pc}$ in the stream reference frame (assuming a Lorentz factor of the stream $\Gamma_{\text {stream }}=10$, and a characteristic recollimation shock length much shorter than the stream length in the host galaxy frame).

Instead, if flares are generated along the path of a superluminal moving perturbation (or knot), the average path length should be $30-60 \mathrm{pc}$ (assuming that the Lorentz factor of the superluminal feature is $\left.\Gamma_{k n o t}=10\right)$. This scenario is not compatible with the $1 / d^{2}$ scaling of the energy density of the magnetic and external photon field in leptonic models (with $d$ the distance of the superluminal feature from the SMBH). The effect on emission of the $1 / d^{2}$ scaling of external fields could be partially mitigated if the superluminal feature accelerates along its path.

Finally the reconnection particle accelerating scenario Giannios 2013) divides the acceleration mechanism in the generation of reconnection events, and in the plasmoid-chain grown within each reconnection event (Sironi, Giannios \& Petropoulou 2016). In this scenario, we should associate the bursting activity to the jet magnetic field instabilities (that generate reconnection events) or to the plasma injection to the reconnection sites, and the fast component to the gamma-ray emission from the plasmoid chain. Thence the

$\overline{1}$ http://www.oato.inaf.it/blazars/webt/ 


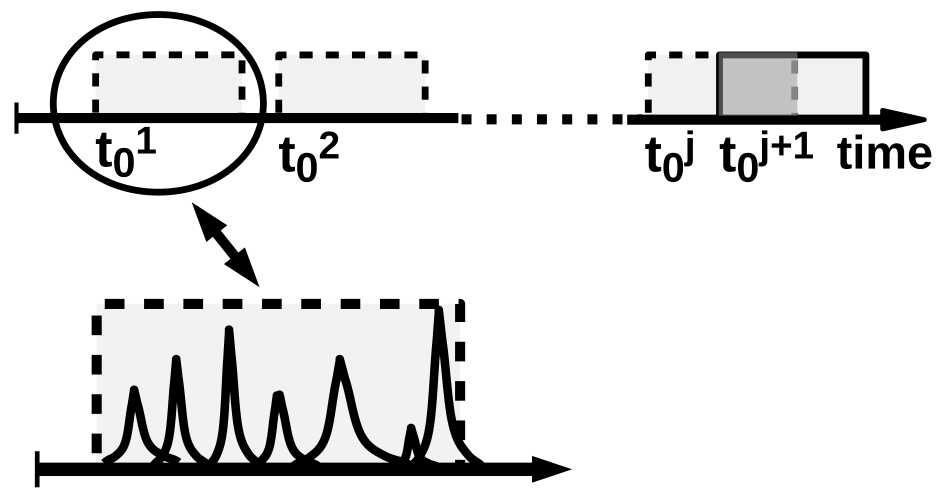

Fig. 10. example of burst activity (multi-hat distribution): each gray rectangle represents a burst of activity. Flares are produced with an uniform distribution inside the bursts of activity. The burst starting time $t_{0}^{j}$ is uniformly distributed along the observing period, the duration of all the bursts is the same. Bursts can overlap.

typical timescale of persistence of a single instability (or of duration of the plasma injection) should be $\sim 0.6 \mathrm{y}$, and the rate of generation of magnetic instabilities (or the rate of plasma injection) should be $\sim 1.3 y^{-1}$. Moreover the typical waiting times between gamma-ray detected plasmoids should be $\sim 1 \mathrm{~h}$.

Several authors (see, e.g., Kelly et al. 2009; Ivezic \& MacLeod 2013) found that Quasars optical variability can be described with a damped random walk, with damping timescale $\tau_{\text {damping }}$ of the order of several hundreds of days. Kelly et al. 2009 also observed that Radio Loud Quasars show an excess optical variability for timescales below $1 \mathrm{~d}$, with a white noise PSD. Recently Burke et al. 2021 found that the damping timescale found in optical correlates with SMBH mass in AGNs: $\tau_{\text {damping }} \sim 110-260 \mathrm{~d}$ for masses in the range of $10^{8}-10^{9} M_{\odot}$.

In our analysis we found similar timescales. This similarity suggests that physical mechanism responsible for optical variability on long timescales could be correlated with the gamma-ray emission process responsible for burst activity of FSRQs.

\section{Appendix A: temporal distributions for flaring times adopted for fittings:}

In this study we make use of several distributions of flaring times:

- An uniform distribution, characterized by the total number of flares $N_{\text {flares }}$.

- an overlapping set of uniformly distributed bursts of activity with all bursts of the same duration $\tau_{\text {burst }}$ (called multi-hat, see fig. 10). Flaring probability is uniform within the burst.

This distribution is characterized by the burst rate parameter $r_{\text {burst }}$, by $\tau_{\text {burst }}$, and $N_{\text {flares }}$;

- an overlapping set of uniformly distributed bursts of activity with $\tau_{\text {burst }}$ distributed with a $\log$ normal distribution (called multi-loghat). Flaring probability is uniform within the burst.

This distribution is characterized by the burst rate parameter $r_{\text {burst }}$, by $N_{\text {flares }}$ and the parameters of the log-normal distribution $\left(m_{\log (\tau)}\right.$ and $\left.\sigma_{\log (\tau)}\right)$.

- an overlapping set of bursts of activity with all bursts of the same duration $\tau_{\text {burst }}$. The distribution of flares within each burst follows a power-law distribution $\left(\rho_{\text {pow }}\right.$, reported in Equation 2),

$\rho_{\text {pow }} \propto \frac{1}{\left|t-t_{0}\right|^{1-\frac{1}{\eta}}} \quad$ with $\eta>0$

around a pole $t_{0}$ conventionally placed at the end of the bursting period.

This distribution is characterized by the burst rate parameter $r_{\text {burst }}$, by $N_{\text {flares }}, \eta$ coefficient, and $\tau_{\text {burst }}$.

This distribution is called multi-pow.

- an overlapping set of bursts of activity, with bursts of duration $\tau_{\text {burst }}$ distributed with a $\log$ normal distribution. The distribution of flares within each burst follows a power-law distribution ( $\rho_{\text {pow }}$, reported in 2), around a pole $t_{0}$ conventionally placed at the end of the bursting period.

This distribution is characterized by the burst rate parameter $r_{\text {burst }}$, by $N_{\text {flares }}, \eta$ coefficient, and the parameters of the $\log$-normal distribution $\left(m_{\log (\tau)}\right.$ and $\left.\sigma_{\log (\tau)}\right)$.

This distribution is called multi-pow-smooth.

- A composite model obtained adding a poissonian process to the multi-loghat fitting function: The event time of a fraction $\mathrm{R}_{\text {fast observed }}$ of the events, is extracted at a time distance $\Delta_{t}$ from an event of the multi-loghat distribution. $\Delta_{t}$ is extracted with an exponential distribution $\rho\left(\Delta_{t}\right)=\frac{e^{\frac{-\Delta_{t}}{e_{\text {fast }}}}}{\tau_{\text {fast }}}$.

This distribution is characterized by the burst rate parameter $r_{\text {burst }}$, by $N_{\text {flares }}$, by the parameters of the log-normal distribution $\left(m_{\log (\tau)}\right.$ and $\left.\sigma_{\log (\tau)}\right)$, and by $R_{\text {fast observed }}, \tau_{\text {fast }}$. This distribution is called multi-loghat + poissonian.

- A composite model obtained adding a poissonian process to the multi-pow fitting function: The event time of a fraction $\mathrm{R}_{\text {fast observed }}$ of the events, is extracted at a time distance $\Delta_{t}$ from an event of the multi-pow distribution. $\Delta_{t}$ is extracted with an exponential distribution $\rho\left(\Delta_{t}\right)=\frac{e^{\frac{-\Delta_{t}}{f_{\text {fast }}}}}{\tau_{\text {fast }}}$.

This distribution is characterized by the burst rate parameter $r_{\text {burst }}$, by $N_{\text {flares }}, \eta$ coefficient, and $\tau_{\text {burst }}$ of the multi-pow distribution, and by $R_{\text {fast observed }}, \tau_{\text {fast }}$.

This distribution is called multi-pow + poissonian.

Fitting method is explained in appendix B.

\section{Appendix B: fitting method}

The realization of the fitting distribution is obtained with a montecarlo method: it is the simulated distribution of waiting times.

It is assumed that waiting times distribution is the same for all the sources, and that only the observed number of flares can vary from source to source.

For each source, we extract the number of emitted bursts within the observing period, their starting time, and length. Finally, 
starting from the observed number of flares for the chosen source, for each flare we extract the burst to which the flare belongs, and the flare starting time within the burst. This last choice corresponds to the assumption that peak flux and duration of each flare does not depend on emission time within a burst. For each source we perform one thousand of simulations. We adopted the binned Cash statistic (Cash 1979) to fit model to the data. There is a chance that a null number of bursts is extracted for a given source. In this case, the simulation cannot reproduce the observed number of flares. So, for each source, the probability to observe a given configuration is obtained by the product of: the probability that the extracted number of burst is not null $\left(1-P_{\text {null }}^{\text {src }}\right)$ and the usual term discussed in Cash (1979). Thence we have to add an element for each source to the Cash estimator; this element is $-2 \ln \left(1-P_{n u l l}^{\text {src }}\right)$.

\section{Appendix C: temporal pile-up effect on the waiting times sample}

Often authors study crowded periods of activity of blazars trying to resolve single flares with a fitting strategy (see, e.g., Abdo et al. 2011; Meyer, Scargle \& Blandford 2019); a predefined flare temporal shape for flares is adopted. Often the shape is parameterized as in equation 7 in Abdo et al. (2010). In this paper we do not make use of this method. Only statistically detected peaks with the iSRS method belongs to the studied sample. During crowded periods of activity, temporal pile-up of flares results in a reduction of the number of detected activity peaks.

Pile-up effect was already addressed in appendix B of Pacciani (2018). Here we compare that findings with simulated samples. We simulated flares with a multi-loghat temporal distribution of flaring times. Once the simulated temporal series were obtained for each source, we performed the $i S R S$ procedure to obtain the flaring peak time, luminosity and duration.

The results for the simulated 100 and $300 \mathrm{MeV}$ samples are summarized in figure 11. We plotted the waiting times versus the minimum among peak fluxes of two consecutive resolved flares $\left(\min \left(F_{i}, F_{i+1}\right)\right.$, where $F_{i}$ and $F_{i+i}$ are the measured peak fluxes of consecutive flares). to facilitate the comparison, in both the plots the reported flux is evaluated for $\mathrm{E}>100 \mathrm{MeV}$ (a power-law index is assumed with index 2.23). The comparison of the two plot reveals that the resolving power is larger for the $100 \mathrm{MeV}$ sample: roughly speaking, the larger statistics allows for an easier separation of close-by flares.

In the same figure, the sensitivity limit is reported for the detection of a flare with temporal FWHM which is the double of the waiting time, and flux corresponding to $\min \left(F_{i}, F_{i+1}\right)$. A curve corresponding to the resolving power of consecutive flares is reported too (see Pacciani 2018, for details).

Acknowledgements. LP thanks IAPS-INAF for support on funds Ricerca di Base F.O. 1.05.01.01, and contribution from the grant INAF Main Stream project High-energy extragalactic astrophysics: toward the Cherenkov Telescope Array, F.O. 1.05.01.86.26. We acknowledge all Agencies and Institutes supporting the Fermi-LAT operations and the Scientific Analysis Tools. L.P. is grateful to Fabrizio Tavecchio for useful suggestions.

\section{References}

Abdo, A. A. e 1., 2010, ApJL, 722, 520;

Abdo, A. A. e 1., 2011, ApJL, 733, 26;

Acero, F., Ackermann, M., Ajello, M., et al., 2015, ApJS, 218, 23;
Aller H. D., Aller M. F., Hughes P. A., 1985, ApJ, 298, 296;

Aschwanden, M. J., McTiernan, J. M., 2010, ApJ, 717, 683;

Atwood, W. B., et al., 2009, ApJ, 697, 1071;

Barnacka, A, Geller, M. J., Dell'Antonio I. P., Zitrin, I., 2016, ApJ, 821, 58; Begelman, M. C., 1998, ApJ, 493, 291;

Biggs, A.D., Browne, I.W.A.,Helbig, P., Koopmans, L.V.E., Wilkonson, P.N., Perley, R.A., 1999, 304, 349:

Blandford R., Eichler D., 1987, Phys. Rev., 154, 1;

Bottcher, M., Reimer, A., Sweeney, K, Prakash, A., 2013, ApJ, 768, 54;

Burke, C., J., et al., 2021, Sci, 373, 789;

Cash, W., 1979, ApJ, 228, 939;

Caprioli, D., Spitkovsky, A., 2014, ApJ, 794, 46;

Casadio, C., et al., 2015, ApJ, 813, 51;

Casadio, C., et al., 2019, A\&A, 622, 158;

Cohen, A. S., Hewitt, J. N., Moore, C. B., Haarsma, D. B., 2000, ApJ, 545, 578;

Celotti, A., Padovani, P., \& Ghisellini, G., 1997, MNRAS, 286, 415

Christie, I. M., Petropoulou, M., Sironi, L., Giannios, D., 2019, MNRAS, 482, 65 ;

D’Ammando F. et al., 2019, MNRAS, 490, 5300;

Dermer, C. D., 1995, ApJ, 446, L63;

Dermer, C. D., Sturner, S. J., Schlickeiser, R., 1997, ApJS, 109, 103;

Felten, J. E., \& Morrison, P., 1966, ApJ, 146, 686;

Fernandes, C. A. C. et al., 2011, MNRAS, 411, 1909;

Frosini, B. V., 1987, "On the distribution and power of a goodness-of-fit statistic with parametric and nonparametric applications". Goodness-of-fit, (Ed. by Revesz P., Sarkadi K., Sen P.K.) Amsterdam-Oxford-New York: NorthHolland, 133-154

Giannios, D., 2013, MNRAS, 431, 355;

Giannios, D., Uzdensky, D. A., 2019, MNRAS, 484, 1378;

Guo F., Li H., DaughtonW., Liu Y. H., 2014, Phys. Rev. Lett., 113, 155005;

Hartigan, J. A., and Hartigan, P. M., 1985, The Annals of Statistics, 13, 70;

Homan, D. C., et al., (2015), ApJ, 798, 134;

Hovatta, T., Valtaoja, E., Tornikoski, M., Lahteenmaki, A., 2009, A\&A, 494, 52;

Hughes, P. A., Aller, H. D., Aller, M. F., 1985, ApJ, 298, 301;

Ivezic, Z., \& macLeod, C., 2013, Proc of the IAU, Cambridge University Press, 9, 395;

Jorstad, S. G., et al., 2017, ApJ, 846, 98;

Kelly, B. M., Bechtold, J., Siemiginowska, A., 2009, ApJ, 698, 895;

Kolmogorov, A.N., 1933, Giornale dell'Instituto Italiano degli Attuari, 4, 83;

Larionov, V. M, et al., 2017, Galaxies, 5, 91;

Larionov, V. M, et al., 2020, MNRAS, 492, 3829L;

Lister, M., L., \& Marscher, A. P., 1997, ApJ, 476, 576;

Lister, M., L., et al., 2013, AJL, 146, 120;

Lister, M., L., et al., 2016, AJL, 152, 12;

Lister, M., L., et al., 2019, ApJ, 874, 43;

Maraschi, L., Ghisellini, G., and Celotti, A., 1992, ApJL, 397, 5;

Marscher, A. P., and Bloom, S. D., 1992, Proceedings of The Compton Observatory Science Workshop, p. 346;

Marscher, A. P., 2013, 2012 Fermi Symposium proceedings -eConf C121028, arXiv:1304.2064

Marscher, A. P., 2014, ApJ, 780, 87;

Meyer, M., Scargle, J. D., Blandford, R. D., 2019, ApJ, 877, 39;

Narayan, R., Piran, T., 2012, MNRAS, 420, 604;

Nagakawa, K., Mori, M., 2013, ApJ, 773, 171;

Pacciani, L., 2014, ApJ, 790, 45;

Pacciani, L., 2018, A\&A, 615, 56;

Pushkarev, A.B., Kovalev, Y. Y., Lister, M. L., Savolainen, T., 2017, MNRAS, 468, 4992;

Raiteri, C. M., et Al., 2021, MNRAS, 501, 1100;

Raiteri, C. M., et Al., 2021, MNRAS, 504, 5629;

Rawling S., Saunders R., 1991, Nat, 349, 138;

Ricker, G. R., et A.L., 2015, Journal of Astronomical Telescopes, Instruments and Systems, 1, 014003;

savolainen, T., et al., 2010, A\&A, 512, 24;

Sironi, L., Keshet, U., Lemoine, M., SSRv, 191, 519;

Sironi, L., Giannios, D., Petropoulou, M., 2016, MNRAS, 462, 3325;

Sobolewska M. A., Siemiginowska A., Kelly B. C., Nalewajko K., 2014, ApJ, 786, 143;

Sbarrato T., Padovani P., Ghisellini G., 2014, MNRAS, 445, 81;

spruit, H. C., Daigne, F., Drenkhahn, G., 2001, A\&A, 369, 694;

Tavecchio, F., Bonnoli, G., Galanti, G., 2020, MNRAS, 497, 1294;

Urry, M., Padovani, P., 1995, PASP, 107, 803;

Wheatland, M. S., 2000, ApJ, 536, 109L;

Wheatland, M. S., Litvinenko, Y. E., 2002, Solar Physics, 211, 255;

Willott C. J., Rawlings S. Jarvis M. J., Blundell K. M., 1999, MNRAS, 309, 1017 ;

Vermeulen, R. C., \& Cohen, M. H., 1994, ApJ, 430, 467;

Villata, M., et Al., 2002, A\&A, 390, 407;

Zenitani S., Hoshino M., 2001, ApJL, 562, 63; 

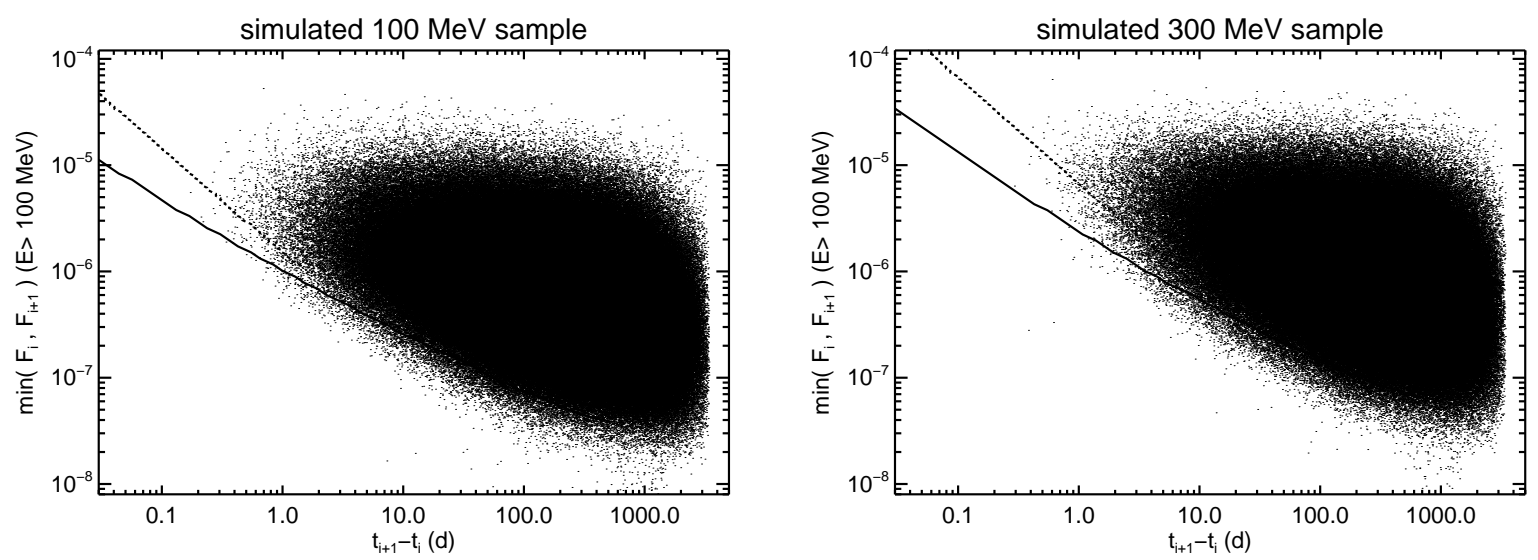

Fig. 11. distribution of $\min \left(F_{i}, F_{i+1}\right)$ vs $t_{i+1}-t_{i}$ for the simulated 100 and $300 \mathrm{MeV}$ samples, left and right plot respectively. For the $300 \mathrm{MeV}$ sample, the flux was scaled to match the flux for gamma-ray above $100 \mathrm{MeV}$ (a power-law flux with photon index of 2.23 is assumed). solid line is the sensitivity limit for a flare from the bright source 3C 454.3 with FWHM twice the waiting times $t_{i+1}-t_{i}$. Dashed line is the temporal resolving power. 